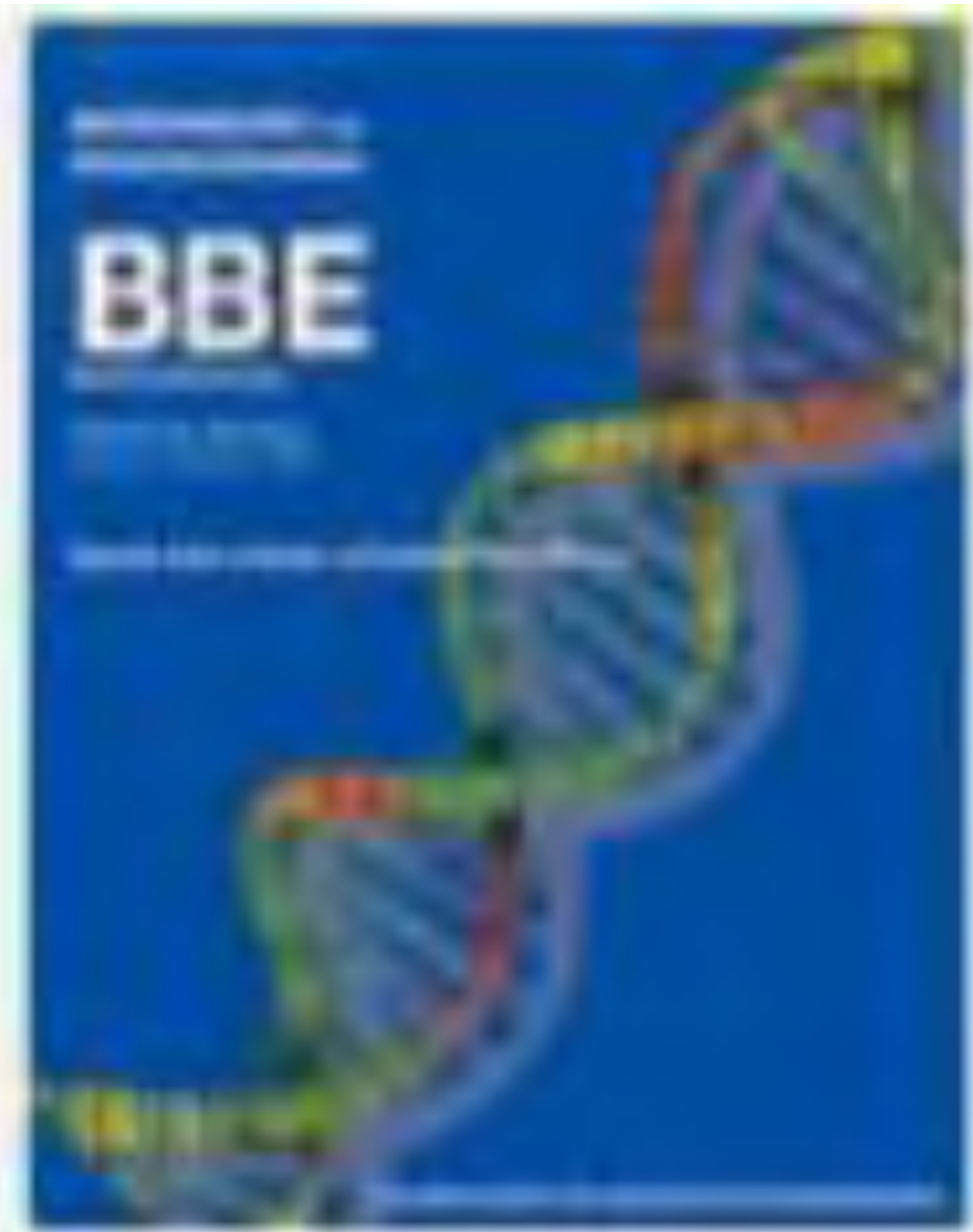




\section{Source details}

\section{Biotechnology and Bioprocess Engineering}

Scopus coverage years: from 1996 to Present

Publisher: Korean Society for Biotechnology and Bioengineering

ISSN: $1226-8372$

Subject area:

Biochemistry, Genetics and Molecular Biology: Biotechnology

Engineering: Biomedical Engineering

Immunology and Microbiology: Applied Microbiology and Biotechnology

Chemical Engineering: Bioengineering
CiteScore 2020

3.6

SJR 2020

0.544

SNIP 2020

0.797 (i)

(i)

(i)

Source type: Journal

\begin{tabular}{|l|l|l|l|l} 
View all documents $>\quad$ Set document alert $\square$ Save to source list
\end{tabular}

CiteScore CiteScore rank \& trend Scopus content coverage

i Improved CiteScore methodology

CiteScore 2020 counts the citations received in 2017-2020 to articles, reviews, conference papers, book chapters and data papers published in 2017-2020, and divides this by the number of publications published in 2017-2020. Learn more >

CiteScore 2020

$$
3.6=\frac{1.417 \text { Citations } 2017-2020}{391 \text { Documents } 2017-2020}
$$

Calculated on 05 May, 2021

CiteScore rank 2020 (i)
Rank Percentile

\begin{tabular}{|c|c|c|}
\hline $\begin{array}{l}\text { Biochemistry, } \\
\text { Genetics and } \\
\text { Molecular Biology } \\
\text { L Biotechnology }\end{array}$ & $\# 133 / 282$ & $53 \mathrm{rd}$ \\
\hline $\begin{array}{l}\text { Engineering } \\
\begin{array}{l}\leftarrow \text { Biomedical } \\
\text { Engineering }\end{array}\end{array}$ & \#111/229 & 5lst \\
\hline
\end{tabular}

\section{CiteScoreTracker 2021 (i)}

$$
4.0=\frac{1.283 \text { Citations to date }}{324 \text { Documents to date }}
$$

Last updated on 04 July, 2021 • Updated monthly

View CiteScore methodology $>$ CiteScore FAQ $>$ Add CiteScore to your site $\mathbb{O}$

$\begin{array}{lll}\text { About Scopus } & \text { Language } & \text { Customer Service } \\ \text { What is Scopus } & \text { 日本語に切り替える } & \text { Help } \\ \text { Content coverage } & \text { 切换到简体中文 } & \text { Contact us } \\ \text { Scopus blog } & \text { 切換到繁體中文 } & \\ \text { Scopus API } & \text { Русский язык } & \\ \text { Privacy matters } & \end{array}$


Terms and conditions $\pi$ Privacy policy $\pi$

Copyright $\left(\right.$ Elsevier B.V $\pi$. All rights reserved. Scopus ${ }^{\circledR}$ is a registered trademark of Elsevier B.V.

We use cookies to help provide and enhance our service and tailor content. By continuing, you agree to the use of cookies. 


\section{Biotechnology and Bioprocess Engineering}

COUNTRY

South Korea

IIII Universities and research
SUBJECT AREA AND CATEGORY

Biochemistry, Genetics and Molecular Biology

- Biotechnology

Chemical Engineering

$\llcorner$ Bioengineering

Engineering

LBiomedical Engineering

Immunology and Microbiology

ᄂApplied Microbiology and Biotechnology

PUBLICATION TYPE

Journals

54

COVERAGE

$1996-2020$
INFORMATION

Homepage

How to publish in this journal

jwyun@daegu.ac.kr
PUBLISHER

Korean Society for Biotechnology and Bioengineering

ISSN

12268372

SCOPE

Biotechnology and Bioprocess Engineering is an international bimonthly journal published by the Korean Society for Biotechnology and Bioengineering. BBE is devoted to the advancement in science and technology in the wide area of biotechnology, bioengineering, and (bio)medical engineering. This includes but is not limited to applied molecular and cell biology, engineered biocatalysis and biotransformation, metabolic engineering and systems biology, bioseparation and bioprocess engineering, cell culture technology, environmental and food biotechnology, pharmaceutics and biopharmaceutics, biomaterials engineering, nanobiotechnology, and biosensor and bioelectronics. 


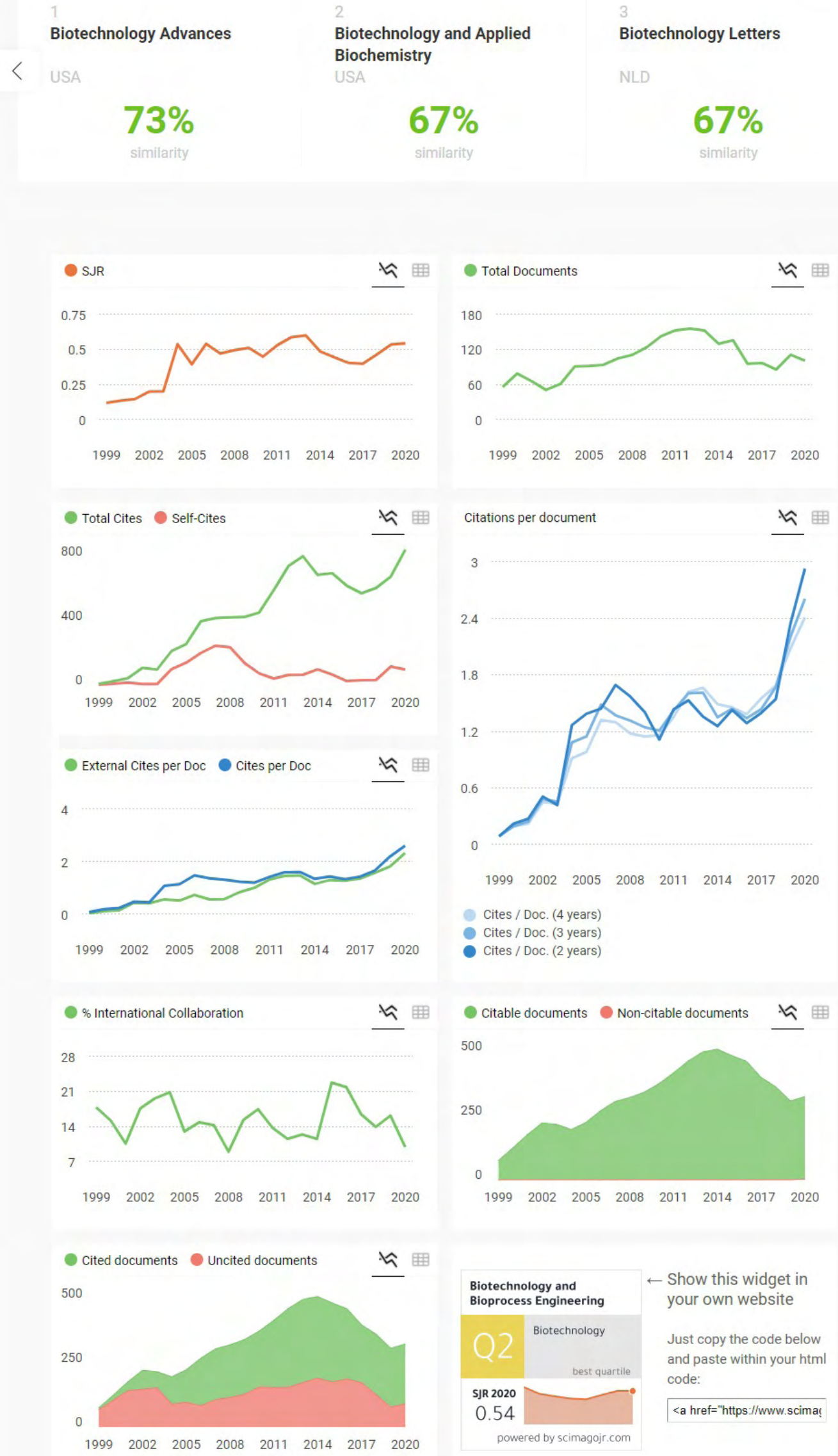




\section{G SClmago Graphica}

Explore, visually

communicate and make sense of data with our new free tool.

Get it

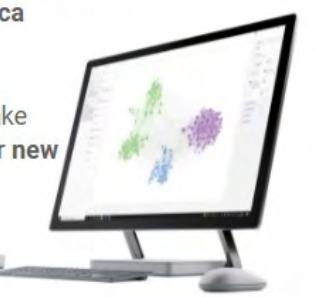

Metrics based on Scopus $\AA^{\circledR}$ data as of April 2021

\section{Leave a comment}

Name

Email

(will not be publishect)

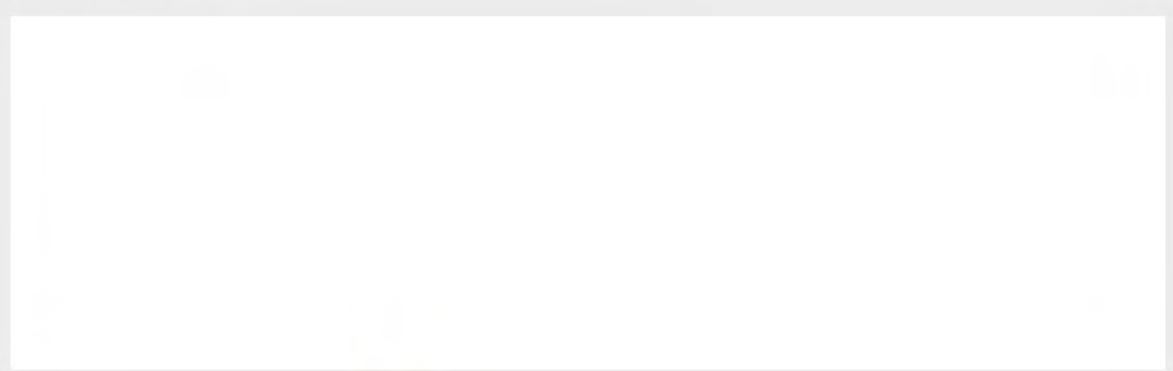

Saya bukan robot

The users of Scimago Journal \& Country Rank have the possibility to dialogue through comments linked to a specific journal. The purpose is to have a forum in which general doubts about the processes of publication in the journal, experiences and other issues derived from the publication of papers are resolved. For topics on particular articles, maintain the dialogue through the usual channels with your editor.

Developed by

(1.) SCImago
Powered by:

Scopus

Follow us on @ScimagoJR

Scimago Lab, Copyright 2007-2020. Data Source: Scopus® 


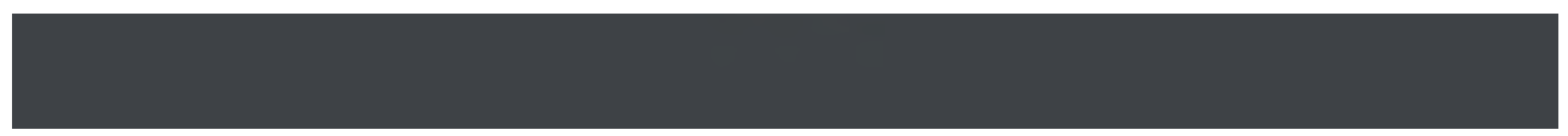




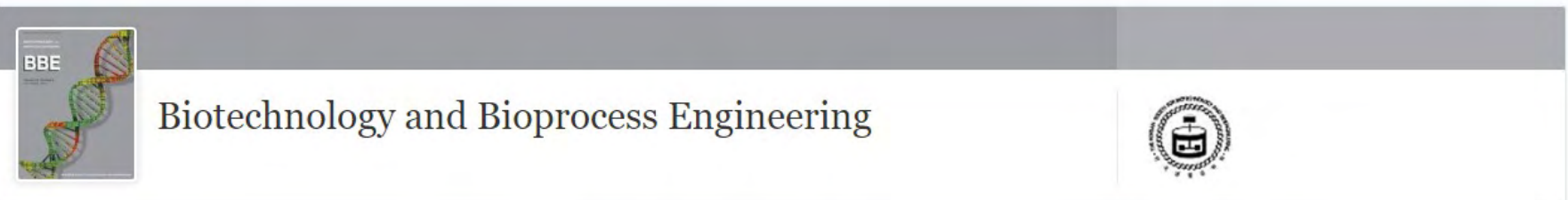

Journal home > Editors

\section{Editors}

\section{Editors-in-Chief}

Jong Won Yun, Ph.D. (Daegu University, Korea)

email: jwyun@daegu.ac.kr

website link: http://mcms.daegu.ac.kr/user/jwyun/

Sang Yup Lee, Ph.D. (Korea Advanced Institute of Science and Technology, Korea) email: leesy@kaist.ac.kr

website link: http://mbel.kaist.ac.kr/lab/index_en.html

\section{International Advisory Board}

Jay D Keasling, Ph.D. (University of California, Berkeley, USA) James Liao, Ph.D. (University of California, Los Angeles, USA) Jens B Nielsen, Ph.D. (Chalmers University of Technology, Sweden)

E. Terry Papoutsakis, Ph.D. (University of Delaware, USA)

Danilo Porro, Ph.D. (University of Milano Bicocca, Italy)

Alexander Steinbüchel, Ph.D. (University of Münster, Germany)

\section{Executive Editors}

Dong-Myung Kim, Ph.D. (Chungnam National University, Korea)

email:dmkim@cnu.ac.kr

Gyoo Yeol Jung, Ph.D. (Pohang University of Science and Technology, Korea) email: gyjung@postech.ac.kr

\section{Editors}

Jong-Sup Bae, Ph.D. (Kyungpook National University, Korea)

Kaustubh D. Bhalerao, Ph.D. (University of Illinois, USA)

Wilfred Chen, Ph.D. (University of Delaware, USA)

Hyung Joon Cha, Ph.D. (Pohang University of Science and Technology, Korea)

Seung-Woo Cho, Ph.D. (Yonsei University, Korea)

Yung Hyun Choi, Ph.D. (Dong-Eui University, Korea)

Suk-Jin Ha, Ph.D. (Kangwon National University, Korea)

Jae-Hyung Jang, Ph.D. (Yonsei University, Korea)

Taek Jin Kang, Ph.D. (Dongguk University, Korea)

Doman Kim, Ph.D. (Seoul National University, Korea)

Yong Hwan Kim, Ph.D. (Ulsan National Institute of Science and Technology, Korea)

Mattheos Koffas, Ph.D. (Rensselaer Polytechnic Institute, USA)

Soonjo Kwon, Ph.D. (Inha University, Korea)

Chang-Soo Lee, Ph.D. (Chungnam National University, Korea)

Jin Woo Lee, Ph.D. (Dong-A University, Korea)

Jeong Geol Na, Ph.D. (Sogang University, Korea)

Min-Kyu Oh, Ph.D. (Korea University, Korea)

Enoch Y. Park, Ph.D. (Shizuoka University, Japan)

Jong Pil Park, Ph.D. (Chung-Ang University, Korea)

Si Jae Park, Ph.D. (Ewha Womans University, Korea)

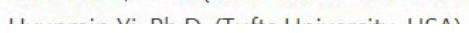

\section{For authors}

Submission guidelines

Ethics \& disclosures

Fees and funding

Contact the journal

\section{Submit manuscript}

\section{Explore}

Online first articles

Volumes and issues

Collections 


\section{Editorial Board}

Qingfeng Chen, Ph.D. (A*STAR, Singapore)

Wei-Chun Chin, Ph.D. (University of California Merced, USA)

Jonghoon Choi, Ph.D. (Chung-Ang University, Korea)

Shin Sik Choi, Ph.D. (Myongji University, Korea)

Yoo Seong Choi, Ph.D. (Chungnam National University, Korea)

Yoon-E Choi, Ph.D. (Korea University, Korea)

Ji-Sook Hahn, Ph.D. (Seoul National University, Korea)

Arum Han, Ph.D. (Texas A\&M University, USA)

Jong Wook Hong, Ph.D. (Hanyang University, Korea)

Ki Yong Hong, M.D., Ph.D. (Seoul National University, Korea)

Yu-Chen Hu, Ph.D. (National Tsing Hua University, Taiwan)

Yun Suk Huh, Ph.D. (Inha University, Korea)

Byeong Hee Hwang, Ph.D. (Incheon National University, Korea)

Dong Soo Hwang, Ph.D. (Pohang University of Science and Technology, Korea)

Nathaniel S. Hwang, Ph.D. (Seoul National University, Korea)

Yu-Sin Jang, Ph.D. (Gyeongsang National University, Korea)

Tae-Joon Jeon, Ph.D. (Inha University, Korea)

Ki Jun Jeong, Ph.D. (Korea Advanced Institute of Science and Technology, Korea)

Jeong Chan Joo, Ph.D. (The Catholic University of Korea, Korea)

Sang Taek Jung, Ph.D. (Korea University, Korea)

Noriho Kamiya, Ph.D. (Kyushu University, Japan)

Eunsung Kan, Ph.D. (Texas A\&M University, USA)

Hyun Kang, M.D., Ph.D. (Chung-Ang University, Korea)

Dae Heon Kim, Ph.D. (Sunchon National University, Korea)

Janghwan Kim, Ph.D. (Korea Research Institute of Bioscience and Biotechnology, Korea)

Jaehong Kim, M.D.,Ph.D. (Gachon University, Korea)

Jung Rae Kim, Ph.D. (Pusan National University, Korea)

Kyobum Kim, Ph.D. (Dongguk University, Korea)

Soo Rin Kim, Ph.D. (Kyungpook National Universi ty, Korea)

Taehyung Kim, Ph.D. (Chung-Ang University, Korea)

Yang-Hoon Kim, Ph.D. (Chungbuk National University, Korea)

Vinod Kumar, Ph.D. (Uttaranchal University, UK)

Dae Hyuk Kweon, Ph.D. (Sungkyunkwan University, Korea)

Inchan Kwon, Ph.D. (Gwangju Institute of Science and Technology, Korea)

Dae Sung Lee, Ph.D. (Kyungpook National University, Korea)

Donghyun Lee, Ph.D. (Chung-Ang University, Korea)

Jeong Wook Lee, Ph.D. (Pohang University of Science and Technology, Korea)

Jintae Lee, Ph.D. (Yeungnam University, Korea)

Min-Woo Lee, Ph.D. (Keimyung University, Korea)

Seung-Wuk Lee, Ph.D. (University of California Berkeley, USA)

Hyejung Mok, Ph.D. (Konkuk University, Korea)

You-Kwan Oh, Ph.D. (Pusan National University, Korea)

Seung Pil Pack, Ph.D. (Korea University, Korea)

Chulhwan Park, Ph.D. (Kwangwoon University, Korea)

Dong Ho Park, M.D., Ph.D. (Kyungpook National University, Korea)

Jason Park, Ph.D. (National Institute of Water and Atmospheric Research, New Zealand)

Tae Jung Park, Ph.D. (Chung-Ang University, Korea)

Hemang Patel, M.D. (Ascension Health, USA)

Won Jong Rhee, Ph.D. (Incheon National University, Korea)

Kalaiselvi Senthil, Ph.D. (Avinashilingam Institute for Home Science and Higher Education for Women,

India)

Sang Woo Seo, Ph.D. (Seoul National University, Korea)

Jung U Shin, M.D., Ph.D. (CHA University, Korea)

Youngsoon Um, Ph.D. (Korea Institute of Science and Technology, Korea)

Han Min Woo, Ph.D. (Sungkyunkwan University, Korea)

Chunping Xu, Ph.D. (Zhengzhou University of Light Industry, China)

Yung Hun Yang, Ph.D. (Konkuk University, Korea)

Tae Hyeon Yoo, Ph.D. (Ajou University, Korea)

Hyungdon Yun, Ph.D. (Konkuk University, Korea)

Feng Zhao, Ph.D. (Institute of Urban Environment Chinese Academy of Sciences, China) 


$\begin{array}{ll}\text { Publish with us } & \text { Discover content } \\ \text { Authors \& Editors } & \text { SpringerLink } \\ \text { Journal authors } & \text { Books A-Z } \\ \text { Publishing ethics } & \text { Journals A-Z } \\ \text { Open Access \& Springer } & \text { Video }\end{array}$

$\begin{array}{ll}\text { Other services } & \text { About Springer } \\ \text { Instructors } & \text { About us } \\ \text { Librarians (Springer Nature) } & \text { Help \& Support } \\ \text { Societies and Publishing } & \text { Contact us } \\ \text { Partners } & \text { Press releases } \\ \text { Advertisers } & \text { Impressum } \\ \text { Shop on Springer.com } & \end{array}$

Legal

General term \& conditions

California Privacy Statement

Rights \& permissions

Privacy

How we use cookies

Manage cookies/Do not sell my data

Accessibility

Not logged in - 36.68.223.177

11741 SpringerLink Indonesia eJourn Consortium (3000951794) - 6763 SpringerLink Indonesia eJourna Consortium - Higher Education (3000122892) - Universitas Surabaya (3000170368)

SPRINGER NATURE

(c) 2021 Springer Nature Switzerland AG. Part of Springer Nature. 


\section{Biotechnology and Bioprocess Engineering}

Journal home > Volumes and issues $>$ volume 24, issue 1

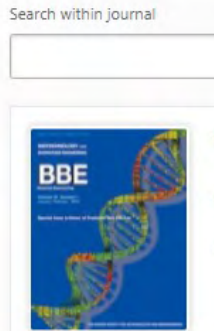

Special Issue to Mark the Career and the Retirement of Professor Sun Bok Lee

Sung Kuk Lee \& Keehoon Won

Editorial | Published: 01 February 2019 | Pages: 1 -2

Celebration of Professor Sun Bok Lee's Pioneering Research Accomplishments and Contributions to the Education of Next Millennial Generation in the Field of Biotechnology and Bioengineering on the Occasion of His Retirement from POSTECH Dewey D. Y. Ryu

A Personal Note | Published: 01 February 2019| Pages: 3 . 7

Molecular-level Antibody Repertoire Profiling and Engineering: Implications for Developing Next-generation Diagnostics, Therapeutics, and Vaccines

Jiwon Lee

Perspective | Published: 01 February $2019 \mid$ Pages: 8-11

Engineering Escherichia coli to Sense Non-native Environmental Stimuli: Synthetic Chimera Two-component Systems

Irisappan Ganesh, Tae Wan Kim ... Soon Ho Hong

Review Paper | Published: 05 January 2019 | Pages: 12 - 22

Microbial Production of Fatty Acid via Metabolic Engineering and Synthetic Biology

Chandran Sathesh.Prabu, Kwang Soo Shin Sung Kuk Lee

Review Paper | Published: 08 February 2019 | Pages: 23 - 40

Esterification of Secondary Alcohols and Multi-hydroxyl Compounds by Candida antarctica Lipase B and Subtilisin

Hee-Jeong Cha, Jin-Byung Park \& Seongsoon Park

Review Paper | Published: 05 January 2019 | Pages: 41 - 47

Recent Advances in the Metabolic Engineering of Klebsiella pneumoniae: A Potential Platform Microorganism for Biorefineries

Mi Na Rhie, Hee Taek Kim ... Si Jae Park

Review Paper | Published: 08 February 2019 | Pages: 48 - 64

A Review of Image Analysis in Biochemical Engineering

Sang-Kyu Jung

Review Paper Published: 29 December 2018 | Pages: 65 -75

P-Protein: A Novel Target for Skin-whitening Agent

Birendra Kumar Singh \& Eun-Ki Kim

Review Paper | Published: 18 February 2019 | Pages: 76 - 84

In vivo Protein Evolution, Next Generation Protein Engineering Strategy: from Random Approach to Target-specific Approach

Jin Young Kim, Hee-Wang Yoo ... Byung-Gee Kim

Review Paper Published: 01 February 2019 Pages: 85 - 94

Effects of gltA and arcA Mutations on Biomass and 1,3-Propanediol Production in Klebsiella pneumoniae
For authors

Submission guidelines

Ethics \& disclosures

Fees and funding

Contact the journal

\section{Submit manuscript}

Explore

Online first articles

Volumes and issues

collections

Sign up for alerts 
Jung Hun, Hwi-Min Jung ... Min-Kyu Oh

Enhanced Incorporation of Gaseous $\mathrm{CO}_{2}$ to Succinate by a Recombinant Escherichia coli W3110

Soohyun Park, Hyeonsoo Kim ... Jinwon Lee

Research Paper | Published: 29 December 2018 | Pages: 103 - 108

Development of Biosensor for 3-Hydroxypropionic Acid

Nam Hoai Nguyen, Jung-Rae Kim \& Sunghoon Park

Research Paper | Published: 05 January 2019 | Pages: 109 - 118

Genomic Characterization of a Newly Isolated Rhizobacteria Sphingomonas panacis Reveals Plant Growth Promoting Effect to Rice

Yeon-Ju Kim, Jaewon Lim ... Deok Chun Yang

Research Paper | Published: 08 February 2019 | Pages: 119 - 125

Beneficial Effects of Desalinated Magma Seawater in Ameliorating Thioacetamideinduced Chronic Hepatotoxicity

Hyunjoo Lee, In Soo Suh... Yeong Ok Song

Research Paper | Published: 08 February 2019 | Pages: 126 - 134

Electrochemical Dopamine Biosensor Composed of Silver Encapsulated $\mathrm{MoS}_{2}$ Hybrid Nanoparticle

Jae-Wook Shin, Jinho Yoon ... Jeong-Woo Cho

Research Paper | Published: 08 February 2019 | Pages: 135 - 144

Development of Cellulose Hydrogel Microspheres for Lipase Immobilization

Soyeon Jo, Saerom Park ... Sang Hyun Lee

Research Paper | Published: 08 February $2019 \mid$ Pages: 145 - 154

Crystal Structure of a Novel Type Isomerase of Enoyl-CoA Hydratase/Isomerase Family Protein from Cupriavidus necator $\mathrm{H} 16$

Hogyun Seo \& Kyung-Jin Kim

Research Paper | Published: 18 February $2019 \mid$ Pages: $155-162$

Apolipoprotein(a) Kringles for Gene Therapy of Colon Cancer

Kyuhyun Lee, Sung-Tae Yun ... Eui-Cheol Jo

Research Paper $\mid$ Published: 08 February 2019 Pages: 163 - 175

Activity Improvements of an Engineered $\omega$-transaminase for Ketones Are Positively Correlated with Those for Cognate Amines

Sang-Woo Han \& Jong-Shik Shin

Research Paper $\mid$ Published: 01 February $2019 \mid$ Pages: 176 - 182

Enhanced Emulsifying and Calcium-binding Properties of Fermented Soybean Meal

Sangkwon Park, Kyung Hoon Chang \& Seong Jun Cho

Research Paper | Published: 05 January 2019 | Pages: 183 - 190

CFD-DEM Simulation of the Fluidized-bed Granulation of Food Powders

Ju-Eun Kim \& Young Mi Chung

Research Paper Published: 29 December 2018 Pages: 191 - 205

Design, Synthesis, and Evaluation of Gold Nanoparticle-Antibody-Horseradish

Peroxidase Conjugates for Highly Sensitive Chemiluminescence Immunoassay (hs-CLIA)

Gyeo-Re Han \& Min-Gon Kim

Research Paper | Published: 08 February $2019 \mid$ Pages: $206-214$

Enzymatic Synthesis of D-pipecolic Acid by Engineering the Substrate Specificity of Trypanosoma cruzi Proline Racemase and Its Molecular Docking Study

Sungmin Byun, Hyun June Park ... Yong Hwan Kim

Research Paper | Published: 18 February 2019 | Pages: 215 - 222

Autotrophic Biodiesel Production from the Thermotolerant Microalga Chlorella sorokiniana by Enhancing the Carbon Availability with Temperature Adjustment

Yoon Young Choi, Min-Eui Hong ... Sang Jun Sim

Research Paper | Published: 01 February 2019 | Pages: 223 - 231

Isolation of Novel CO Converting Microorganism Using Zero Valent Iron for a

Bioelectrochemical System (BES)

Hveon Suna Im. Chanaman Kim ... Juna Rae Kim 
Fluorescence-based Quantification of Bioactive Keratin Peptides from Feathers for Optimizing Large-scale Anaerobic Fermentation and Purification

Hyeon-Su Jin, Seon Yeong Park ... Dong-Woo Lee

Research Paper | Published: 01 February 2019 | Pages: 240 - 249

Isolation of Novel Exo-type $\beta$-Agarase from Gilvimarinus chinensis and High-level Secretory Production in Corynebacterium glutamicum

Yong Jun Jeong, Jae Woong Choi ... Ki Jun Jeong

Research Paper | Published: 01 February $2019 \mid$ Pages: $250-257$

Wood Powder as a New Natural Sunscreen Ingredient

Sang Cheon Lee, Sang Hyun Lee \& Keehoon Won

Research Paper | Published: 18 February 2019 | Pages: 258 - 263

Prescreening of Natural Products in Drug Discovery Using Recombinant Bioluminescent Bacteria

Eui Jong Kim, Ho Bin Seo \& Man Bock Gu

Research Paper | Published: 01 February 2019 | Pages: 264 - 271

Home $\mid$ Impressum $\mid$ Legal information $\mid$ Privacystatement $\mid$ California Privacy Statement $\mid$ How we use cookies $\mid$ Manage cookies/Do not sell my data $\mid$ Accessibility $\mid \underline{\text { Contact us }}$

Not logged in - 36.68.223.177

11741 SpringerLink Indonesia elourn Consortium (3000951794) - 6763 SpringerLink Indonesia eJouma Consortium - Higher Education (3000122392) - Universtas Surabaya (3000170368)

SPRINGER NATURE

2021 Springer Nature Switzerland AG. Part of Şpringer Nature 


\title{
Genomic Characterization of a Newly Isolated Rhizobacteria Sphingomonas panacis Reveals Plant Growth Promoting Effect to Rice
}

\author{
Yeon-Ju Kim, Jaewon Lim, Johan Sukweenadhi, Ji Woong Seok, Sang-Won Lee, Jong Chan Park, \\ Assiya Taizhanova, Donghyuk Kim, and Deok Chun Yang
}

Received: 9 October 2018 / Revised: 17 October 2018 / Accepted: 17 October 2018

(C) The Korean Society for Biotechnology and Bioengineering and Springer 2019

\begin{abstract}
This article reports the full genome sequence of Sphingomonas panacis $\mathrm{DCY}^{\mathrm{T}} 9^{\mathrm{T}}\left(=\mathrm{KCTC} \quad 42347^{\mathrm{T}}\right.$ $=\mathrm{JCM} 30806^{\mathrm{T}}$ ), which is a Gram-negative rod-shaped, nonspore forming, motile bacterium isolated from rusty ginseng root in South Korea. A draft genome of S. panacis DCY99 and a single circular plasmid were generated using the PacBio platform. Antagonistic activity experiment showed $S$. panacis $\mathrm{DCY} 99^{\mathrm{T}}$ has the plant growth promoting effect. Thus, the genome sequence of $S$. panacis $\mathrm{DCY} 9^{\mathrm{T}}$ may contribute to biotechnological application of the genus Sphingomonas in agriculture.
\end{abstract}

Keywords: Sphingomonas panacis, genome, plant growth promoting rhizobacteria (PGPR), systemic resistance, Xanthomonas oryzae

Yeon-Ju Kim ${ }^{\dagger *}$, Jaewon Lim $†$, Johan Sukweenadhi, Sang-Won Lee, Jong Chan Park, Assiya Taizhanova, Deok Chun Yang

Graduate School of Biotechnology, College of Life Science, Kyung Hee University, Yongin 17104, Korea

Tel: +82-31-201-2645; Fax: +82-31-204-8116

E-mail: yeonjukim@khu.ac.kr

Jaewon Lim, Assiya Taizhanova, Donghyuk Kim*

School of Energy and Chemical Engineering, Ulsan National Institute of

Science and Technology (UNIST), Ulsan 44919, Korea

Tel: +82-52-217-2945; Fax: +82-52-217-3009

E-mail:dkim@unist.ac.kr

Ji Woong Seok

Lab Genomics Co. Ltd, Seongnam 13487, Korea

Donghyuk Kim

School of Biological Sciences, Ulsan National Institute of Science and Technology (UNIST), Ulsan 44919, Korea

Donghyuk Kim

Korean Genomics Industrialization and Commercialization Center, Ulsan National Institute of Science and Technology (UNIST), Ulsan 44919, Korea

'These authors contributed equally.

\section{Introduction}

The genus Sphingomonas was first identified by Yabuuchi et al. [1]. Sphingomonas is classified within the family Sphingomonadaceae, the order Sphingomonadales and the a-4 group of the class Alpha-proteobacteria. Takeuchi et al. proposed the genus Sphingomonas sensu stricto and three new genera, Sphingobium, Novosphingobium, and Sphingopyxis, on the basis of phylogenetic and chemotaxonomic analyses [2,3]. Species in the genus Sphingomonas are diverse and can adapt to various environments such as bacterial growth plates [4], bulk/sea/waste water [5-7], alpine soil [8], spacecrafts [9], and arctic-lichen [10].

Members of the genus Sphingomonas are traditionally known as degraders well adapted for the bioremediation of polycyclic aromatic hydrocarbons [8,9,11,12]. Many strains of this genus can produce exopolysaccharides such as sphingans [13] and welan [13-16]. Some members of this group have been described as plant growth-promoting bacteria [17-20].

Panax ginseng C. A. Meyer is one of the most widely used herbal medicinal plants in Korea. Sphingomonas species were detected on the root surface of rusty ginseng by culture-dependent and culture-independent methods combined with metagenome analysis. The isolated novel strains Sphingomonas panacis $\mathrm{DCY} 99^{\mathrm{T}}$ and S. panaciterrae DCY91 showed antagonist activity against the severe ginseng pathogen Ilyonectria and also elicited systemic resistance in rice to its pathogen, Xanthomonas oryzae. Here we report the whole genome sequence and circular mega plasmid of strain $S$. panacis isolated from rusty ginseng root. The genome sequence of $S$. panacis DCY99 ${ }^{\mathrm{T}}$ provides a better understanding of its genetic background for more effective utilization of this strain. 


\section{Materials and Methods}

\subsection{Isolation and culture conditions}

S. panacis DCY $99^{\mathrm{T}}$ was isolated from soil of a ginseng field in Hwacheon province, Republic of Korea by serial dilution method. S. panacis DCY99 ${ }^{\mathrm{T}}\left(\right.$ KCTC $42347^{\mathrm{T}}=$ JCM $30806^{\mathrm{T}}$ ) was grown in DSMZ medium 1 (Nutrient Agar) at $28^{\circ} \mathrm{C}$.

\subsection{Genome sequencing and assembly}

The genomic DNA was isolated using JetFlex Genomic DNA purification kit (ThermoFisher). The extracted DNA was used to generate $20 \mathrm{~kb}$ SMRTbell $^{\mathrm{TM}}$ template libraries. Genome sequencing was performed at DNA Link, Inc. using the Pacific Biosciences RSII sequencing method. A draft genome of $S$. panacis $\mathrm{DCY} 99^{\mathrm{T}}$ was generated using de novo Hierarchical Genome Assembly Process (HGAP) implemented within the analysis pipeline SMRT Analysis 2.2 (Pacific Biosciences, CA, USA).

The draft genome sequence has been deposited in the NCBI (BioProject PRJNA308882), Genbank IDs are CP014168 for the main chromosome and CP014169 for the plasmid.

\subsection{Gene annotation}

Open reading frames (ORFs) were predicted using Glimmer 3.02 and predicted genes were annotated using Blastall 2.2.26. tRNA and rRNA genes were identified by tRNAscanSE version 1.3 and RNAmmer version 1.2. Protein coding genes were also analyzed with the Clusters of Orthologous Groups of proteins (COGs) database.

\subsection{Phylogenetic tree analysis}

21 Sphingomonas strains with genomic sequences were selected for a phylogenetic tree analysis. The phylogenetic tree was constructed by using 16S rRNA gene sequences with Clustal Omega (https://www.ebi.ac.uk/Tools/msa/clustalo/) [21].

\subsection{Comparative genomic analysis}

NCBI BLAST alignment tool (blastn) was used to align multiple genome sequences. BLAST was performed using default parameters and an e-value of 10 , mismatch penalty of -3 , and matching reward of 1 . The genome and plasmid were visualized by the comparison using the Artemis software and ACT.

\subsection{Antagonistic activity experiment}

The top agar method was used to determine the in vitro antagonistic activity of the Sphingomonas strains towards Xoo PXO99Az. The size of the halo zone is used as an efficiency of antagonistic activity. Xoo PXO61 was used as a negative control. The in vivo antagonistic activity was measured following. Xoo PXO99Az strain was grown on peptone sucrose agar ( $1 \%$ peptone, $1 \%$ sucrose, $0.1 \%$ glutamic acid, and $1.5 \%$ bacto agar, $\mathrm{pH} 7.5$, PSA) containing cephalexin $(15 \mathrm{mg} / \mathrm{L})$ for 3 days, suspended in distilled water at approximately $1.0 \times 109 \mathrm{CFU} / \mathrm{ml}$ and then inoculated using the clipping method. S. panaciterrae DCY91 and $S$. panacis DCY99 were streaked on TSA medium. All 3 strains incubated at $28^{\circ} \mathrm{C}$ for 3 days. After incubation, strains were harvested and suspended in distilled water when the optical density at $600 \mathrm{~nm}$ reached 2.0. The tips of TP309 leaves were clipped, and the leaves were placed in the bacterial solutions and allowed to soak for $24 \mathrm{~h}$. Lesion development was monitored for two weeks. Three different samples were measured as biological replicates.

\section{Results and Discussion}

\subsection{Classification and features}

The type strain, S. panacis DCY $99^{\mathrm{T}}$ was isolated from soil of a ginseng field in Hwacheon province, Republic of Korea by serial dilution method. The results of metal tolerating capability of $S$. panacis $\mathrm{DCY} 99^{\mathrm{T}}$ are corresponding to the its sampling origin (rusty ginseng roots). There was a significant amount of heavy metals accumulated in the rusty surface of ginseng. Classification and general features of $S$. panacis DCY $99^{\mathrm{T}}$ were described in Table 1 . The previous result showed that $S$. panacis $\mathrm{DCY} 99^{\mathrm{T}}$ is Gramnegative, aerobic, non-spore forming, motile, rod shaped, oxidase and catalase positive [19]. The overall shape of S. panacis $\mathrm{DCY} 99^{\mathrm{T}}$ was analyzed with scanning electron microscope $S$. panacis (Fig. 1A). Colonies are circular, entire, low convex, smooth, opaque, light yellow, and 0.1$1.0 \mathrm{~mm}$ in diameter after growth on nutrient agar (NA) plates for 1 day. The bacteria grow optimally at $25-30^{\circ} \mathrm{C}$ and $\mathrm{pH}$ 6.0-6.5. Phylogenetic tree analysis with 16S rRNA gene sequences highlighted the taxonomic position of S. panacis $\mathrm{DCY} 99^{\mathrm{T}}$ and $S$. panaciterrae $\mathrm{DCY}^{\mathrm{T}}{ }^{\mathrm{T}}$ within the genus Sphingomonas (Fig. 1B). The isoprenoid quinone detected was ubiquinone Q-10 and sym-homospermidine as the major polyamine. The major polar lipids were sphingoglycolipid, diphosphatidylglycerol, phosphatidylethanolamine, phosphatidylglycerol and phosphatidylcholine. The major fatty acids were $\mathrm{C}_{14: 0} 2 \mathrm{OH}, \mathrm{C}_{16: 0}$ and summered feature $8\left(\mathrm{C}_{18: 1} \omega 7 c: \mathrm{C}_{18: 1} \omega 6 c\right)$. The novel species Sphingomonas panacis $\mathrm{DCY} 99^{\mathrm{T}}$ was registered as the type strain $\left(=\mathrm{JCM} 30806^{\mathrm{T}}=\right.$ KCTC $\left.42347^{\mathrm{T}}\right)$.

\subsection{Growth conditions and genomic DNA preparation} S. panacis $\mathrm{DCY} 99^{\mathrm{T}}$ was selected for genome sequencing because this species produces compounds that protect plants from biotic stress and has growth-promoting activity in rice 
Table 1. Classification and general features of S. panacis DCY99 ${ }^{\mathrm{T}}$

\begin{tabular}{|c|c|c|c|}
\hline MIGS ID & Property & Term & Evidence \\
\hline & Classification & Domain Bacteria & TAS \\
\hline & & Phylum Proteobacteria & TAS \\
\hline & & Class Alphaproteobacteria & TAS \\
\hline & & Order Sphingomonadales & TAS \\
\hline & & Family Sphingomonadaceae & TAS \\
\hline & & Genus Sphingomonas & TAS \\
\hline & & Species Sphingomonas panacis & TAS \\
\hline & & Strain DCY $99^{\mathrm{T}}$ & TAS [19] \\
\hline & Gram stain & Negative & IDA \\
\hline & Cell shape & Rod & IDA \\
\hline & Motility & Motile & IDA \\
\hline & Sporulation & No spore production & IDA \\
\hline & Temperature range & $10-37^{\circ} \mathrm{C}$ & IDA \\
\hline & Optimum temperature & $25^{\circ} \mathrm{C}$ & IDA \\
\hline & $\mathrm{pH}$ range; Optimum & $5.5-7.5 ; 6.0-6.5$ & IDA \\
\hline & Carbon source & Glucose, arabinose, mannose, others & TAS [19] \\
\hline MIGS-6 & Habitat & Mountain cultured rusty Panax ginseng C.A. Meyer root & IDA \\
\hline MIGS-6.3 & Salinity & $0.5-4.5 \% \mathrm{NaCl}$ & IDA \\
\hline MIGS-22 & Oxygen requirement & Aerobic & IDA \\
\hline MIGS-15 & Biotic relationship & Free-living & IDA \\
\hline MIGS-14 & Pathogenicity & Non-pathogenic & NAS \\
\hline MIGS-13 & Source material identifiers & KCTC $42347^{\mathrm{T}}$, JCM $30806^{\mathrm{T}}$ & TAS [19] \\
\hline MIGS-4 & Geographic location & South Korea: Gangwon province & IDA \\
\hline MIGS-5 & Sample collection & April 2013 & IDA \\
\hline MIGS-4.1 & Latitude & $38.153 \mathrm{~N}$ & IDA \\
\hline MIGS-4.2 & Longitude & $127.770 \mathrm{~W}$ & IDA \\
\hline MIGS-4.4 & Altitude & $461 \mathrm{~m}$ & IDA \\
\hline
\end{tabular}

*Evidence codes - IDA Inferred from Direct Assay, TAS, Traceable Author Statement (i.e., a direct report exists in the literature), NAS Non-traceable Author Statement (i.e., not directly observed for the living, isolated sample, but based on a generally accepted property for the species, or anecdotal evidence). These evidence codes are from the Gene Ontology project [24].

and Panax ginseng C. A. Meyer. S. panacis DCY99 $\left(\mathrm{KCTC} 42347^{\mathrm{T}}=\mathrm{JCM} 30806^{\mathrm{T}}\right)$ was grown in DSMZ medium 1 (Nutrient Agar) at $28^{\circ} \mathrm{C}$. DNA was isolated from 0.5-1 $\mathrm{g}$ of cell paste using the JetFlex Genomic purification kit as recommended by the manufacturer. A draft genome of $S$. panacis DCY $99^{\mathrm{T}}$ was generated using the PacBio platform following the manufacturer's instructions.

\subsection{Genome sequencing and assembly}

A total of 96,529 reads with an average length of $10,519 \mathrm{bp}$ were generated and assembled using the de novo Hierarchical Genome Assembly Process (HGAP) implemented within the analysis pipeline SMRT Analysis 2.2 (Pacific Biosciences, CA, USA) (Table S1). Ambiguous bases and inserted/deleted regions between PacBio assembled and preassembled draft sequences were corrected manually by using consensus sequences for final assembly. Long reads were selected as seed sequences for constructing preassemblies, while short reads were mapped to the seeds using BLASTR software for alignment, which corrected errors in the long reads and thus increased the accuracy rating of bases. The sequencing run yielded 1,015,409,853 filtered sub-read bases and a total of 85,634,367 preassembled bases were used for deep sequencing. tRNA and rRNA genes were identified by tRNAscan-SE version 1.3 [22] and RNAmmer version 1.2 [23]. Open reading frames (ORFs) were predicted using Glimmer 3.02 and predicted genes were annotated using Blastall 2.2.26. Protein coding genes were annotated to The Clusters of Orthologous Groups of proteins (COGs) database [24]. Artemis software was used for data management and ACT was used for genome and plasmid visualization [25]. Sequencing project information was summarized (Table 1). The draft genome consisted of a single circular chromosome of 5,003,808 bp with a GC content of $65.66 \%$, which is characteristic of most Sphingomonas strains (60 to 68\%). A total of 4,800 genes were predicted to be encoded by the genome (Table S2). There was a single circular plasmid of 319,133 bp with a GC content of $62.71 \%$ (Table S3).

The draft genome sequence has been deposited in the 

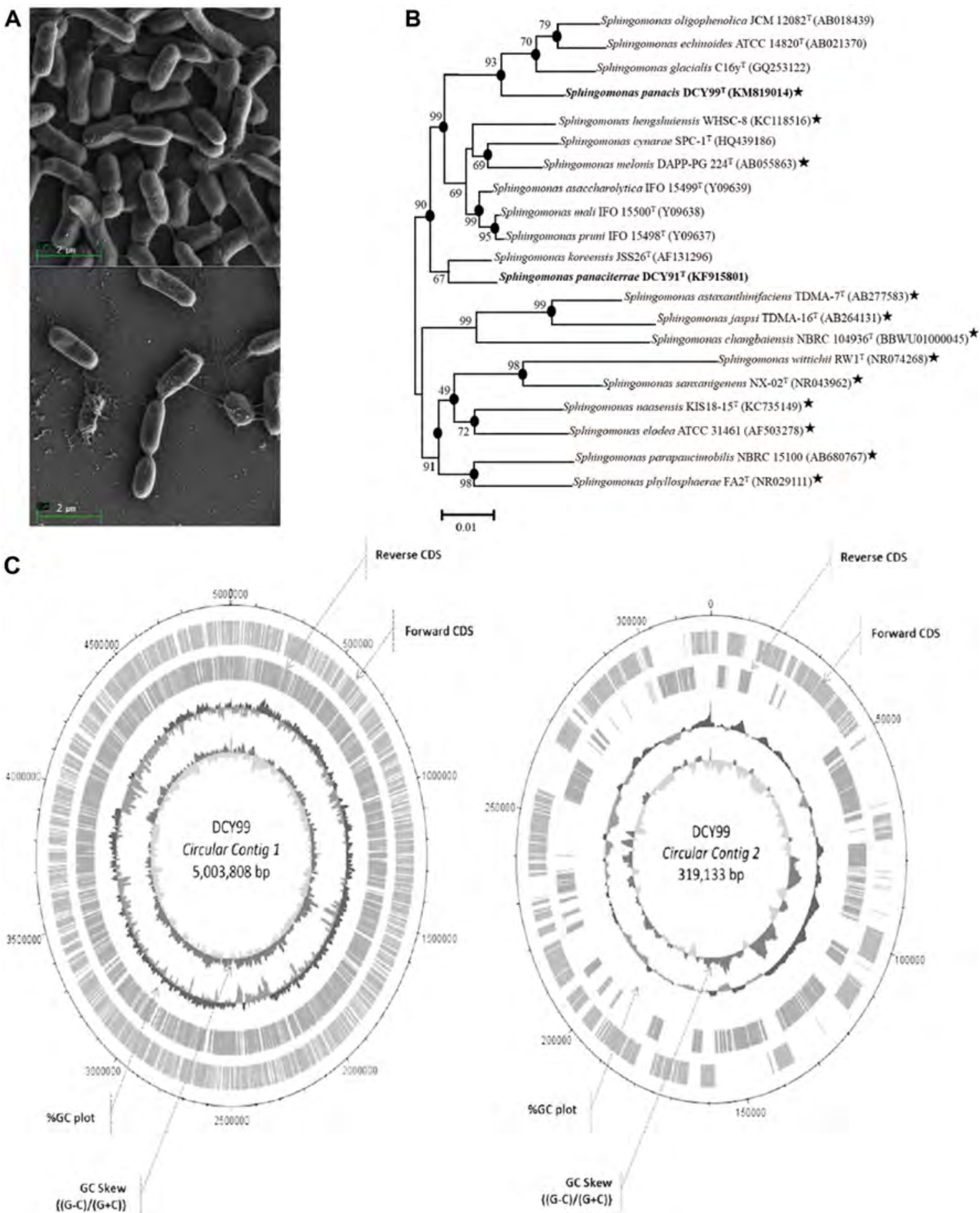

Fig. 1. Morphological and genomic characterization of a newly sequenced $S$. panacis DCY $99^{\mathrm{T}}$. (A) Scanning electron microscope photos of $S$. panacis DCY $99^{\mathrm{T}}$. (B) Phylogenetic tree analysis with $16 \mathrm{~S}$ rRNA gene sequences highlights the taxonomic positions of $S$. panacis DCY $99^{\mathrm{T}}$ and S. panaciterrae DCY91 in the genus Sphingomonas. GenBank accession numbers are indicated in parentheses. Filled circles indicate that the corresponding nodes were also recovered in the tree generated with the neighbor-joining and maximumparsimony algorithms. Stars indicate strains for which draft whole genome sequences are available. Bar, 0.01 substitutions per nucleotide position. (C) Graphical circular map and genetic features of $S$. panacis DCY $99^{\mathrm{T}}$. The genomic features of $S$. panacis DCY $99^{\mathrm{T}}$ for the main chromosome (Contig 1) and the plasmid (Contig 2) are shown. From outside to the center: Genes on the forward strand, genes on the reverse strand, $\mathrm{G}+\mathrm{C}$ content, and GC skew.

NCBI (BioProject PRJNA308882). The genome can be found under BioSample SAMN04417200, and the Genbank
ID is CP014168 for the genome and CP014169 for the plasmid, respectively. 
Table 2. Genome statistics.

\begin{tabular}{lrr}
\hline \multicolumn{1}{c}{ Feature category } & $\begin{array}{c}\text { Genomic } \\
\text { feature }\end{array}$ & Percentile \\
\hline Genome size (bp) & $5,003,808$ & 100.00 \\
DNA coding (bp) & $4,376,256$ & 87.46 \\
DNA G+C (bp) & $3,285,696$ & 65.66 \\
DNA scaffolds & 2 & - \\
Total genes & 4,872 & 100.00 \\
Protein coding genes & 4,722 & 96.92 \\
RNA genes & 62 & 12.73 \\
Pseudo genes & 88 & 18.06 \\
Genes in internal clusters & 1,274 & 26.15 \\
Genes with function prediction & 4,431 & 90.95 \\
Genes assigned to COGs & 3,378 & 69.34 \\
Genes with Pfam domains & 2,039 & 41.85 \\
Genes with signal peptides & 600 & 12.32 \\
Genes with transmembrane helices & 889 & 18.25 \\
CRIPSR repeats & 2 & - \\
\hline
\end{tabular}

\subsection{Genome annotation}

The deposited sequences that both CP014168 and CP014169 were annotated by the NCBI using the Prokaryotic Genome Annotation Pipeline. Genome annotation revealed 4,872 coding sequences (Table 2). The circular visualization of the genome annotation for the main chromosome and the plasmid is presented (Fig. 1C). The COG functional analysis of the genomic DNA of strain S. panacis DCY99 ${ }^{\mathrm{T}}$ was also performed (Fig. S1). Interestingly, genes for indole-3-acetic acid synthesis are present in the genome of S. panacis DCY99 ${ }^{\mathrm{T}}$. These genes corroborate that these S. panacis strains could promote plant growth and elicit systemic resistance. Protein coding genes were annotated to The Clusters of Orthologous Groups of proteins was presented in Table S2 and S3. The genome annotation and the sequences of genes can be used for applications, such as developing detection kits for plant growth promoting bacteria or plant pathogens [26].

\subsection{Insights from the genome sequence}

Genome analysis showed that $S$. panacis $\mathrm{DCY} 99^{\mathrm{T}}$ contained many genes encoding oxidoreductases, chaperones proteins, and metal transporters. The genes encode the enzymes for the synthesis of the nucleotide sugars (UDP-Glc and UDPGlcA) from Glc-1-phosphate such as $p g m G$ and $u g p G$ were found, indicating its ability to produce extracellular polysaccharide sphingans. Several important quorum sensing factor, such as $r s h$ and $l u x R$ were also present on the genome. These genes presumably allow this strain to cope with different environmental stresses. S. panacis DCY $99^{\mathrm{T}}$ might have the potential for application in industrial biotechnology as a producer of miscellaneous hydrolases.

\subsection{Comparative genomic analysis}

NCBI BLAST alignment tool (blastn) was used to align multiple genome sequences. BLAST was performed using default parameters and an e-value of 10 , mismatch penalty of -3 , and matching reward of 1 . The genome and plasmid were visualized by the comparison using the Artemis software and ACT. The genome of $S$. panacis DCY $99^{\mathrm{T}}$ was compared with $S$. wittichii RW1, S. melonis FR1, and S. sanxanigenens NX02, respectively (Fig. 2A). The principal features of the plasmid sequences for bacteria of the genus Sphingomonas were compared (Fig. 2B) Comparison of the genomic sequences available for bacteria within the genus Sphingomonas were summarized in Table S4. Principal features and comparison of the plasmid sequences available for bacteria in the genus Sphingomonas also was presented in Table S5.

\subsection{Sphingomonas enhances resistance to bacterial leaf blight}

Two Sphingomonas strains were tested against the rice
A

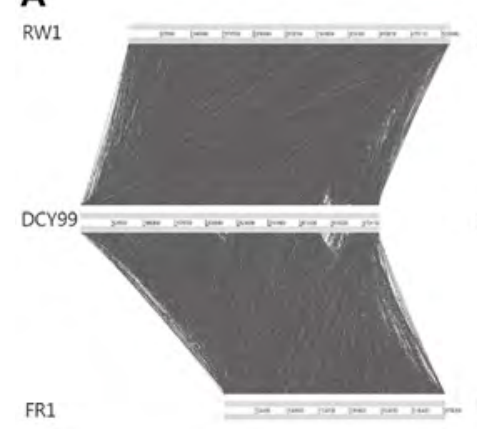

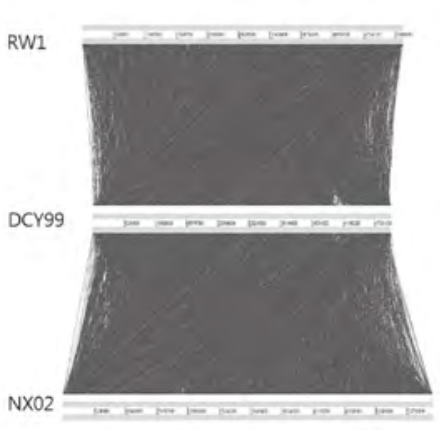

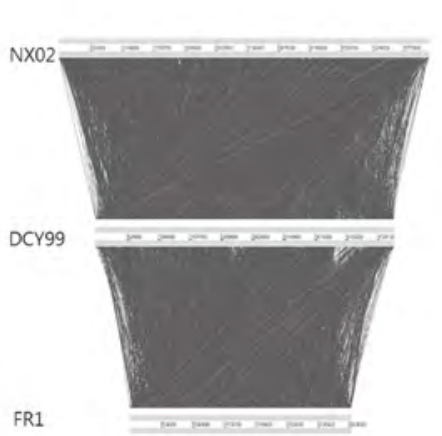

Fig. 2. Comparative genomic analysis for multiple Sphingomonas strains. (A) Genomic sequence comparison analysis for the main chromosomes of $S$. panacis DCY $99^{\mathrm{T}}$, S. wittichii RW1, S. melonis FR1, and S. sanxanigenes NX02. (B) Genomic sequence comparison analysis for the plasmids of $S$. wittichii RW1 (CP000701) and S. sanxanigenens NX02 (CP011450). 
A

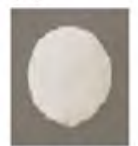

Xoo PX061

B
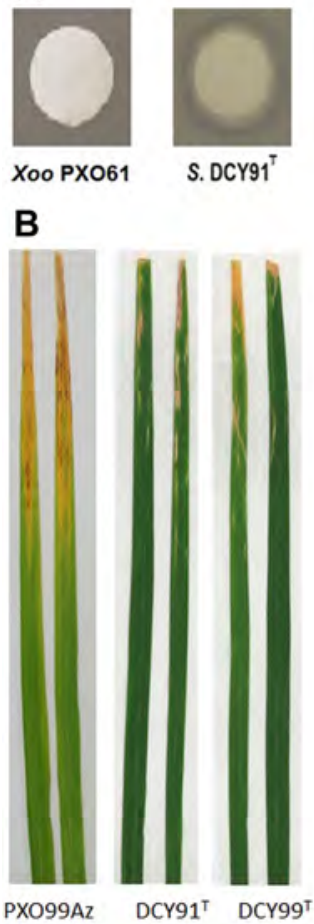

S. DCY $91^{\top}$
S. DCY99

C

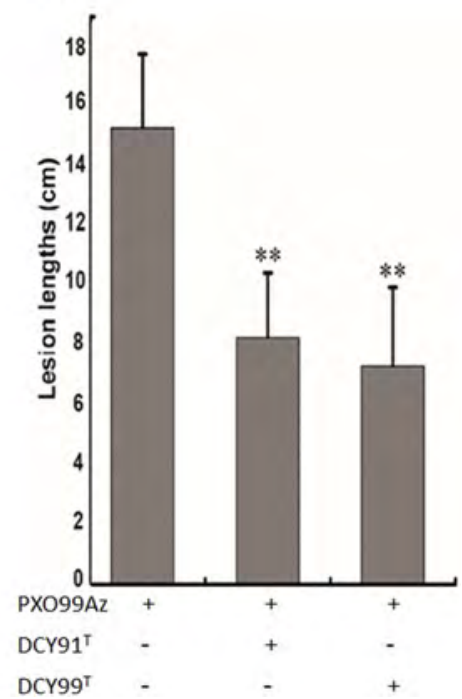

Fig. 3. Evaluation of the antagonistic activity of Sphingomonas against Xoo PXO99Az. (A) In vitro antagonistic test against Xoo PXO99Az. Growth of three bacterial strains, namely Xoo PXO61, $S$. panaciterrae DCY91, and $S$. panacis DCY99 ${ }^{\mathrm{T}}$, on TSA medium containing Xoo PXO99Az was monitored. Eighteen biological replicates were performed. All experiments were repeated three times. (B) Lesion development of two Sphingomonas strain feeding lines and wild-type TP309 inoculated with Xoo PXO99Az. Photograph of rice leaves 14 days after inoculation. (C) Lesion lengths of rice leaves measured 14 days after inoculation. All experiments were repeated three times and the error bars indicate means $\pm \operatorname{SD}(n=9)$. At least three biological replicates were performed. Asterisk indicates $\mathrm{p}<0.01$ (Duncan test). Different letters above bars indicate statistically significant differences as determined by one-way analysis of variance (ANOVA), $\mathrm{p}<0.01$.

pathogenic bacteria Xoo PXO99Az. The top agar method was used to determine the antagonistic activity of the Sphingomonas strains towards Xoo PXO99Az. Presence of a halo zone around a disc was taken as evidence of antagonistic activity (Fig. 3A). S. panaciterrae $\mathrm{DCY} 91^{\mathrm{T}}$ had an antagonistic effect on Xoo PXO99Az as evidenced by a clear halo zone $(2.537 \pm 0.474 \mathrm{~mm})$. The strain $S$. panacis DCY $99^{\mathrm{T}}$ had a greater antibacterial effect on Xoo PXO99Az $(5.722 \pm 0.521 \mathrm{~mm})$ than $S$. panaciterrae DCY91 ${ }^{\mathrm{T}}$. In contrast, the negative control Xoo PXO61 did not have significant antagonistic activity toward Xoo PXO99Az.

S. panaciterrae $\mathrm{DCY} 91^{\mathrm{T}}$ and $S$. panacis $\mathrm{DCY} 99^{\mathrm{T}}$ were streaked on TSA medium and incubated at $28^{\circ} \mathrm{C}$ for 3 days. After incubation, strains were harvested and suspended in distilled water when the optical density at $600 \mathrm{~nm}$ reached
2.0. The tips of TP309 leaves were clipped, and the leaves were placed in the bacterial solutions and allowed to soak for $24 \mathrm{~h}$. Xoo PXO99Az strain was grown on peptone sucrose agar ( $1 \%$ peptone, $1 \%$ sucrose, $0.1 \%$ glutamic acid, and $1.5 \%$ bacto agar, $\mathrm{pH} 7.5$, PSA) containing cephalexin $(15 \mathrm{mg} / \mathrm{L})$ for 3 days, suspended in distilled water at approximately $1.0 \times 10^{9} \mathrm{CFU} / \mathrm{ml}$ and then inoculated using the clipping method. Lesion development was monitored for two weeks. Three different samples were measured as biological replicates. Lesion lengths on rice leaves (TP309) inoculated with two strains (S. panaciterrae DCY91 and S. panacis $\mathrm{DCY}^{\mathrm{T}}{ }^{\mathrm{T}}$ ) were 46 to $53 \%$ lower than rice leaves not inoculated with Sphingomonas (Fig. 3B, Fig. 3C). These data support that the enhanced resistance seen in TP309 was caused by the antagonistic effects of Sphingomonas against bacterial leaf blight.

\section{Conclusion}

This is the first report describing the genome sequence of S. panacis $\mathrm{DCY} 99^{\mathrm{T}}$. The genome size of strain $S$. panacis DCY $99^{\mathrm{T}}(5.0 \mathrm{Mb})$ is smaller than that of other sequenced members of the genus Sphingomonas, including S. sanxanigenens $\mathrm{NX} 02(6.58 \mathrm{Mb})$ and $S$. wittichii RW1 $(5.92 \mathrm{Mb})$. Strain $S$. panacis $\mathrm{DCY} 9^{\mathrm{T}}$ has a $\mathrm{G}+\mathrm{C}$ content $(65.66 \%)$ higher than that of S. paucimobilis EPA505 (63.9\%). Antagonistic activity experiment showed S. panacis DCY $99^{\mathrm{T}}$ has the plant growth promoting effect. Thus, the genome sequence of $S$. panacis $\mathrm{DCY} 99^{\mathrm{T}}$ may contribute to biotechnological application of the genus Sphingomonas in agriculture.

\section{Acknowledgments}

We appreciated to the company, Korea Bird Wild Ginseng Co, in Namyangju, South Korea for collection of the mountain cultured ginseng. This study was supported by a grant from the Cooperative Research Program for Agriculture Science and Technology Development (Project No. PJ0128132017) Rural Development Administration in Republic of Korea, and was also supported by the 2018 Research Fund (1.180074.01) of UNIST (Ulsan National Institute of Science and Technology). YJK, and JL designed the study, carried out the genome analysis, and drafted the manuscript, JS performed DNA isolation, electron microscopy, and the phylogenetic analysis for taxonomic study. JWS, JL, DK, and AT carried out the sequencing and helped to draft the manuscript. SWL and JCP participated in rice in vivo assay. DCY coordinated. All authors read and approved the final manuscript. 
Electronic Supplementary Material (ESM) The online version of this article (doi: 10.1007/s12257-018-0386-2) contains supplementary material, which is available to authorized users.

\section{References}

1. Yabuuchi, E., Y. Kosako, N. Fujiwara, T. Naka, I. Matsunaga, H. Ogura, and K. Kobayashi (2002) Emendation of the genus Sphingomonas Yabuuchi et al. 1990 and junior objective synonymy of the species of three genera, Sphingobium, Novosphingobium and Sphingopyxis, in conjunction with Blastomonas ursincola. International Journal of Systematic and Evolutionary Microbiology 52: 1485-1496.

2. Takeuchi, M., K. Hamana, and A. Hiraishi (2001) Proposal of the genus Sphingomonas sensu stricto and three new genera, Sphingobium, Novosphingobium and Sphingopyxis, on the basis of phylogenetic and chemotaxonomic analyses. International Journal of Systematic and Evolutionary Microbiology 51: 14051417.

3. Glaeser, S. P. and P. Kämpfer (2014) The family sphingomonadaceae. pp. 641-707. The Prokaryotes. Springer, City.

4. Shin, S. C., S. J. Kim, D. H. Ahn, J. K. Lee, and H. Park (2012) Draft genome sequence of Sphingomonas echinoides ATCC 14820. Journal of Bacteriology 194: 1843-1843.

5. Yoon, J.-H., C.-H. Lee, S.-H. Yeo, and T.-K. Oh (2005) Sphingopyxis baekryungensis sp. nov., an orange-pigmented bacterium isolated from sea water of the Yellow Sea in Korea. International Journal of Systematic and Evolutionary Microbiology 55: 1223-1227.

6. Lu, P., C. Chen, Q. Wang, Z. Wang, X. Zhang, and S. Xie (2013) Phylogenetic diversity of microbial communities in real drinking water distribution systems. Biotechnology and Bioprocess Engineering 18: 119-124.

7. Yang, Q., J. Wang, X. Han, Y. Xu, D. Liu, H. Hao, X. Li, Y. Guo, T. Niu, and S. Qi (2014) Analysis of the bacterial community in a full-scale printing and dyeing wastewater treatment system based on T-RFLP and 454 pyrosequencing. Biotechnology and Bioprocess Engineering 19: 191-200.

8. Margesin, R., D.-C. Zhang, and H.-J. Busse (2012) Sphingomonas alpina sp. nov., a psychrophilic bacterium isolated from alpine soil. International Journal of Systematic and Evolutionary Microbiology 62: 1558-1563.

9. Pan, L., H. Zhou, J. Li, B. Huang, J. Guo, X.-L. Zhang, L.-C. Gao, C. Xu, and C.-T. Liu (2016) Draft genome sequence of Sphingomonas paucimobilis strain LCT-SP1 isolated from the Shenzhou X spacecraft of China. Standards in Genomic Sciences 11: 18.

10. Lee, H., S. C. Shin, J. Lee, S. J. Kim, B.-K. Kim, S. G. Hong, E. H. Kim, and H. Park (2012) Genome sequence of Sphingomonas sp. strain PAMC 26621, an Arctic-lichen-associated bacterium isolated from a Cetraria sp. Journal of Bacteriology 194: 30303030 .

11. Ohta, H., R. Hattori, Y. Ushiba, H. Mitsui, M. Ito, H. Watanabe, A. Tonosaki, and T. Hattori (2004) Sphingomonas oligophenolica sp. nov., a halo-and organo-sensitive oligotrophic bacterium from paddy soil that degrades phenolic acids at low concentrations. International Journal of Systematic and Evolutionary Microbiology 54: 2185-2190.
12. Aylward, F. O., B. R. McDonald, S. M. Adams, A. Valenzuela, R. A. Schmidt, L. A. Goodwin, T. Woyke, C. R. Currie, G. Suen, and M. Poulsen (2013) Comparison of 26 sphingomonad genomes reveals diverse environmental adaptations and biodegradative capabilities. Applied and Environmental Microbiology AEM. 00518-00513.

13. Wu, M., G. Li, H. Huang, S. Chen, Y. Luo, W. Zhang, K. Li, J. Zhou, and T. Ma (2016) The simultaneous production of sphingan Ss and poly (R-3-hydroxybutyrate) in Sphingomonas sanxanigenens NX02. International Journal of Biological Macromolecules 82: 361-368.

14. Gai, Z., X. Wang, X. Zhang, F. Su, X. Wang, H. Tang, C. Tai, F. Tao, C. Ma, and P. Xu (2011) Genome sequence of Sphingomonas elodea ATCC 31461, a highly productive industrial strain of gellan gum. Journal of Bacteriology 193: 7015-7016.

15. Wang, X., F. Tao, Z. Gai, H. Tang, and P. Xu (2012) Genome sequence of the welan gum-producing strain Sphingomonas sp. ATCC 31555. Journal of Bacteriology 194: 5989-5990.

16. Tala, A., M. Lenucci, A. Gaballo, M. Durante, S. M. Tredici, D. A. Debowles, G. Pizzolante, C. Marcuccio, E. Carata, and G. Piro (2013) Sphingomonas cynarae sp. nov., a proteobacterium that produces an unusual type of sphingan. International Journal of Systematic and Evolutionary Microbiology 63: 72-79.

17. García-Romero, I., A. J. Pérez-Pulido, Y. E. González-Flores, F. Reyes-Ramírez, E. Santero, and B. Floriano (2016) Genomic analysis of the nitrate-respiring Sphingopyxis granuli (formerly Sphingomonas macrogoltabida) strain TFA. BMC Genomics 17: 93.

18. Wachowska, U., W. Irzykowski, M. Jędryczka, A. D. StasiulewiczPaluch, and K. Głowacka (2013) Biological control of winter wheat pathogens with the use of antagonistic Sphingomonas bacteria under greenhouse conditions. Biocontrol Science and Technology 23: 1110-1122.

19. Singh, P., Y.-J. Kim, V.-A. Hoang, M. E.-A. Farh, and D.-C. Yang (2015) Sphingomonas panacis sp. nov., isolated from rhizosphere of rusty ginseng. Antonie Van Leeuwenhoek 108: 711-720.

20. Sukweenadhi, J., Y.-J. Kim, C. H. Kang, M. E.-A. Farh, N.-L. Nguyen, V.-A. Hoang, E.-S. Choi, and D.-C. Yang (2015) Sphingomonas panaciterrae sp. nov., a plant growth-promoting bacterium isolated from soil of a ginseng field. Archives of Microbiology 197: 973-981.

21. Sievers, F. and D. G. Higgins (2014) Clustal omega. Current Protocols in Bioinformatics 48: 3.13. 11-13.13. 16.

22. Lowe, T. M. and S. R. Eddy (1997) tRNAscan-SE: a program for improved detection of transfer RNA genes in genomic sequence. Nucleic Acids Research 25: 955.

23. Lagesen, K., P. Hallin, E. A. Rødland, H.-H. Stærfeldt, T. Rognes, and D. W. Ussery (2007) RNAmmer: consistent and rapid annotation of ribosomal RNA genes. Nucleic Acids Research 35: 3100-3108.

24. Ashburner, M., C. A. Ball, J. A. Blake, D. Botstein, H. Butler, J. M. Cherry, A. P. Davis, K. Dolinski, S. S. Dwight, and J. T. Eppig (2000) Gene Ontology: tool for the unification of biology. Nature Genetics 25: 25.

25. Carver, T. J., K. M. Rutherford, M. Berriman, M.-A. Rajandream, B. G. Barrell, and J. Parkhill (2005) ACT: the Artemis comparison tool. Bioinformatics 21: 3422-3423.

26. Yun, M., Y.-K. Oh, R. Praveenkumar, Y.-S. Seo, and S. Cho (2017) Contaminated bacterial effects and qPCR application to monitor a specific bacterium in Chlorella sp. KR-1 culture. Biotechnology and Bioprocess Engineering 22: 150-160.

Publisher's Note Springer Nature remains neutral with regard to jurisdictional claims in published maps and institutional affiliations. 